\title{
Introduction to the Anisotropic Geometrodynamics
}


Editor-in-charge: Louis H. Kauffman (Univ. of Illinois, Chicago)

The Series on Knots and Everything: is a book series polarized around the theory of knots. Volume 1 in the series is Louis H Kauffman's Knots and Physics.

One purpose of this series is to continue the exploration of many of the themes indicated in Volume 1. These themes reach out beyond knot theory into physics, mathematics, logic, linguistics, philosophy, biology and practical experience. All of these outreaches have relations with knot theory when knot theory is regarded as a pivot or meeting place for apparently separate ideas. Knots act as such a pivotal place. We do not fully understand why this is so. The series represents stages in the exploration of this nexus.

Details of the titles in this series to date give a picture of the enterprise.

Published*:

Vol. 1: Knots and Physics (3rd Edition)

by L. H. Kauffman

Vol. 2: How Surfaces Intersect in Space - An Introduction to Topology (2nd Edition) by J. S. Carter

Vol. 3: Quantum Topology edited by L. H. Kauffman \& R. A. Baadhio

Vol. 4: Gauge Fields, Knots and Gravity by J. Baez \& J. P. Muniain

Vol. 5: Gems, Computers and Attractors for 3-Manifolds by S. Lins

Vol. 6: Knots and Applications edited by L. H. Kauffman

Vol. 7: Random Knotting and Linking edited by K. C. Millett \& D. W. Sumners

Vol. 8: Symmetric Bends: How to Join Two Lengths of Cord by R. E. Miles

Vol. 9: Combinatorial Physics by T. Bastin \& C. W. Kilmister

Vol. 10: Nonstandard Logics and Nonstandard Metrics in Physics by W. M. Honig

Vol. 11: History and Science of Knots edited by J. C. Turner \& P. van de Griend 
Vol. 12: Relativistic Reality: A Modern View

edited by J. D. Edmonds, Jr.

Vol. 13: Entropic Spacetime Theory

by J. Armel

Vol. 14: Diamond - A Paradox Logic

by N. S. Hellerstein

Vol. 15: Lectures at KNOTS '96

by S. Suzuki

Vol. 16: Delta - A Paradox Logic

by N. S. Hellerstein

Vol. 17: Hypercomplex Iterations - Distance Estimation and Higher Dimensional Fractals by Y. Dang, L. H. Kauffman \& D. Sandin

Vol. 18: The Self-Evolving Cosmos: A Phenomenological Approach to Nature's Unity-in-Diversity by S. M. Rosen

Vol. 19: Ideal Knots

by A. Stasiak, V. Katritch \& L. H. Kauffman

Vol. 20: The Mystery of Knots - Computer Programming for Knot Tabulation by C. N. Aneziris

Vol. 21: LINKNOT: Knot Theory by Computer by S. Jablan \& R. Sazdanovic

Vol. 22: The Mathematics of Harmony — From Euclid to Contemporary Mathematics and Computer Science

by A. Stakhov (assisted by S. Olsen)

Vol. 23: Diamond: A Paradox Logic (2nd Edition)

by N. S. Hellerstein

Vol. 24: Knots in HELLAS '98 - Proceedings of the International Conference on Knot Theory and Its Ramifications

edited by C. McA Gordon, V. F. R. Jones, L. Kauffman, S. Lambropoulou \& J. H. Przytycki

Vol. 25: Connections - The Geometric Bridge between Art and Science (2nd Edition) by J. Kappraff

Vol. 26: Functorial Knot Theory - Categories of Tangles, Coherence, Categorical Deformations, and Topological Invariants by David N. Yetter

Vol. 27: Bit-String Physics: A Finite and Discrete Approach to Natural Philosophy by H. Pierre Noyes; edited by J. C. van den Berg

Vol. 28: Beyond Measure: A Guided Tour Through Nature, Myth, and Number by J. Kappraff

Vol. 29: Quantum Invariants - A Study of Knots, 3-Manifolds, and Their Sets by T. Ohtsuki

Vol. 30: Symmetry, Ornament and Modularity by $S$. V. Jablan 
Vol. 31: Mindsteps to the Cosmos by G. S. Hawkins

Vol. 32: Algebraic Invariants of Links by J. A. Hillman

Vol. 33: Energy of Knots and Conformal Geometry by J. O'Hara

Vol. 34: Woods Hole Mathematics - Perspectives in Mathematics and Physics edited by N. Tongring \& R. C. Penner

Vol. 35: BIOS - A Study of Creation by H. Sabelli

Vol. 36: Physical and Numerical Models in Knot Theory edited by J. A. Calvo et al.

Vol. 37: Geometry, Language, and Strategy by G. H. Thomas

Vol. 38: Current Developments in Mathematical Biology edited by K. Mahdavi, R. Culshaw \& J. Boucher

Vol. 39: Topological Library Part 1: Cobordisms and Their Applications edited by S. P. Novikov \& I. A. Taimanov

Vol. 40: Intelligence of Low Dimensional Topology 2006 edited by J. Scott Carter et al.

Vol. 41: Zero to Infinity: The Fountations of Physics by $P$. Rowlands

Vol. 42: The Origin of Discrete Particles by T. Bastin \& C. Kilmister

Vol. 43: The Holographic Anthropic Multiverse by R. L. Amoroso \& E. A. Ranscher

Vol. 44: Topological Library Part 2: Characteristic Classes and Smooth Structures on Manifolds edited by S. P. Novikov \& I. A. Taimanov

Vol. 45: Orbiting the Moons of Pluto Complex Solutions to the Einstein, Maxwell, Schrödinger and Dirac Equations by E. A. Rauscher \& R. L. Amoroso

Vol. 46: Introductory Lectures on Knot Theory edited by L. H. Kauffman, S. Lambropoulou, S. Jablan \& J. H. Przytycki

Vol. 47: Introduction to the Anisotropic Geometrodynamics by S. Siparov 
RE Series on Knots and Everything - Vol. 47

\section{Introduction to the}
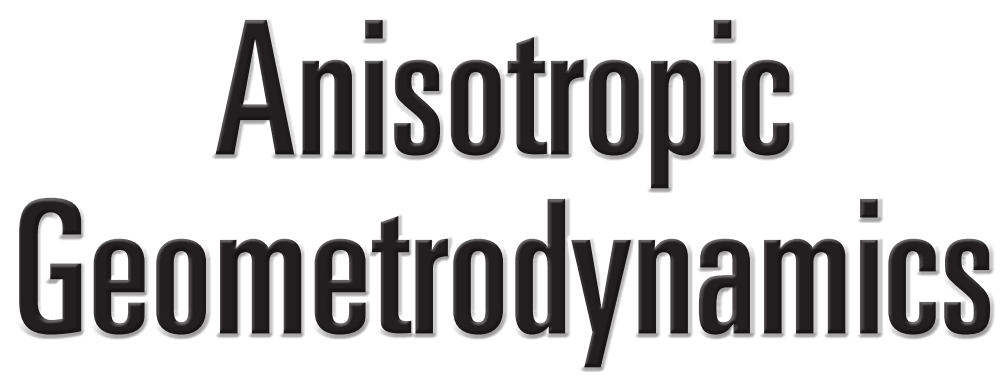

\section{Sergey Siparov}

State University of Civil Aviation, Russia

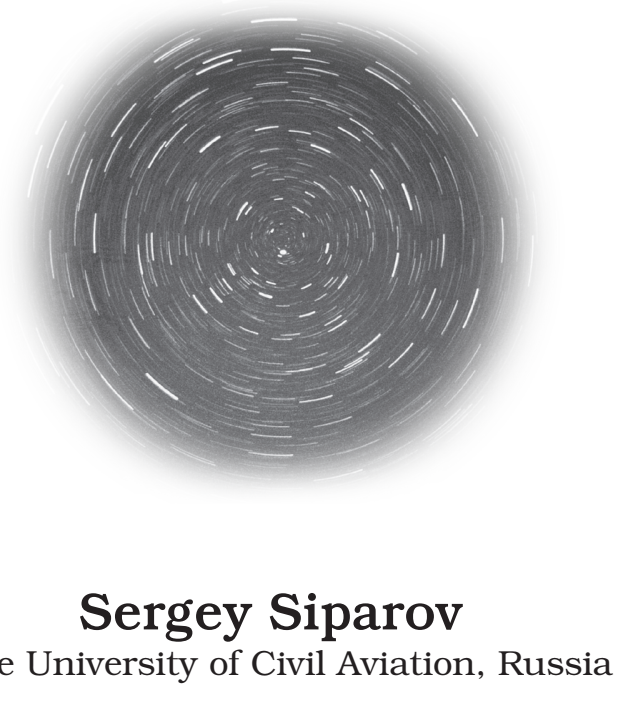




\section{Published by}

World Scientific Publishing Co. Pte. Ltd.

5 Toh Tuck Link, Singapore 596224

USA office: 27 Warren Street, Suite 401-402, Hackensack, NJ 07601

UK office: 57 Shelton Street, Covent Garden, London WC2H 9HE

\section{British Library Cataloguing-in-Publication Data}

A catalogue record for this book is available from the British Library.

\section{INTRODUCTION TO THE ANISOTROPIC GEOMETRODYNAMICS \\ Series on Knots and Everything - Vol. 47}

Copyright (C) 2012 by World Scientific Publishing Co. Pte. Ltd.

All rights reserved. This book, or parts thereof, may not be reproduced in any form or by any means, electronic or mechanical, including photocopying, recording or any information storage and retrieval system now known or to be invented, without written permission from the Publisher.

For photocopying of material in this volume, please pay a copying fee through the Copyright Clearance Center, Inc., 222 Rosewood Drive, Danvers, MA 01923, USA. In this case permission to photocopy is not required from the publisher.

ISBN-13 978-981-4340-83-0

ISBN-10 981-4340-83-9

Printed in Singapore. 


\section{Preface}

Ptolemy was probably the first who designed a world machine that corresponded to what everyone saw and was based on some inherent ideas, but at the same time the mechanism was supplied with details that needed long and skillful observations. There were stars, Sun, Moon and planets that were obviously rotating around the Earth - along the circular orbits belonging to the heavenly spheres, but it turned out that from time to time the planets performed retrograde motions, and Ptolemy set inside various tilted epicycles.

For some time it worked smoothly, but then the bug started to manifest itself in such a way that the errors were accumulating with time. For Copernicus it became clear that the drawback was general. Still, the obvious must have been preserved, and its presentation should have been based on some inherent ideas, while the rest of the device should have been redesigned. The components were reshuffled: Sun took the central position, and the Earth, planets and stars started to rotate around it along circular orbits. It looked good but worked even worse than before, until Kepler noticed and revealed the new details - those planetary orbits were elliptical. This was implemented, and since then the machine seemed to work perfectly. But then the new engineers became curious, why it was so.

Galileo paid attention to the surrounding conditions and proclaimed that the working principles one might be looking for should be independent of place, time and straight uniform motion. These notions required a language with the help of which the strict definitions, demands and instructions could be formulated. And Newton performed this linguistic task and, in parallel, recognized that the independence on straight uniform motion could be achieved only if the reason for the motion is proportional not to the motion itself but to its change. He gave the short definition that became 
the foundation. Turning back to the world machine in possession of new knowledge, he discovered the law on the base of which it was functioning. The discouraging news was that there was a distant force acting between Sun and planets (stars were too far), but nothing else than to remain logically consistent could be done, and Newton had to attribute a universal character to this force.

The next magnificent linguistic achievement was made when they thought of the materials used for the device. One short phrase consisting of four parts pronounced by Maxwell not only perfectly displayed all the huge variety of electromagnetic phenomena, but, unexpectedly, revealed the deep relationship between electromagnetism and optics - that is explained the way we see. And then the thunderbolt came. The light did not obey the velocity addition rule following from the main principle of independence proclaimed by Galileo and known as "relativity principle".

Einstein accepted the blow and stood up with the realization that we see what we see - any time, any place and any straight uniform motion, and that the guesses should be dismissed however clever they are. When we see, it takes time for light to pass a path in space, therefore, the obtained images correspond not to the current positions of objects but to the abandoned positions, and if we want to know how it works, we should focus not on the objects themselves but on the processes involving their motions and light travel times. This approach preserved the Maxwell's achievement and produced the new one: the account for the light travel time added a new dimension to the world picture, and now the motions became not the trajectories on the flat plane governed by the distant forces, but the curved trajectories nested on the relief. The heavenly (and other) bodies produced rather potentials than forces, and these potentials produced the relief. This caused the problem with measuring details of the machine with the help of triangles: the triangle plane did not fit the curved surface. But already Newton was keen enough in regarding infinitely small parts of everything, since then they learned the ways, and Einstein made geometry indistinguishable from dynamics.

The residuary trouble is distances. Currently, we can neither penetrate into the regions of matter where electrodynamics is not enough nor reach the distant parts of the observable Universe. But the signals come from both regions, and we have some nice patterns for their interpretation. The golden dream is to unify the patterns, and the Einstein's rule "we see what we see" seems to be the most appropriate base for that.

This book is a single step in this direction. The need for the large 
amount of "dark notions" that appeared in astronomy in the last decades means that the machine seems to start coughing again. The introduction of those performed in order to make the mechanism fit the drawn specification makes one suspicious. It comes to mind to mimic Einstein's approach, change the point of view and try another model for the background of everything we could observe and register, i.e. for the space and time. A simple analogue of such possible model is known in general physics, it is the phase space. It cannot be perceived by our senses immediately, but it is not hard to get used to it. Such transfer resembles the pass from the Lagrange approach to the Hamilton one, and any physicist is aware of both. The results of the suggested description led to the simplification of the current world picture: there is no need for the dark matter and, possibly, no need for the dark energy in the explanation of the paradoxes in the known observations on the cosmological scale. At the same time all the achievements of the classical GRT on the planetary scale are preserved. In order to suggest a specific test for this modified theory, we need an effect on galactic scale, and it seems that the effect of optic-metrical parametric resonance will do. The effect is not widely known, the first promising observations were performed only recently, and the explanation of its theory requires some technical details. But the result is simple: we can notice the effect of the gravitational radiation of the periodical sources like short-period binary stars with the help of regular radio telescopes, because the spectra of some space masers obtain specific periodic components. A couple of the suitable radio sources has already been found and their signals studied. When the data statistics of such sources is sufficient, it would be possible to investigate the geometry of our galaxy and compare the results with the suggested approach.

Both special and general relativity theories are simple in their deep essence as it usually is with the fundamentals, they are really physical, despite the mathematical entourage that one has to overcome in order to understand them. Being a "land of possibilities" mathematics is very fascinating and meaningful especially when it comes to interpretations. That is why it could sometimes tempt one to go slightly too far and enjoy mirages - images that are surely related to certain physical properties and that are also "seen by everyone" - rather than other wonders of the real world that could appear none the less charming when it comes to the direct testing. Aren't these "other wonders" also mirages but on the deeper level, will be hardly ever known. But this book is a humble attempt to bring relativity closer to the general physics to which it belongs and to focus on the short and simple ideas discovered by Newton, Maxwell and Einstein. 
It's an honor and pleasure to mention those whose efforts made it possible for this book to appear. The idea to write it was suggested by Peter Rowlands and supported by Louis Kauffman. Nicoleta Voicu and Vladimir Samodurov were my collaborators in obtaining some of the results. The essentials and general ideas were many times discussed with Sergey Kokarev, Michail Babich, Alexander Kazakov and Nickolay Razumovsky and also with colleagues from RI HSGP and its head (and soul) Dmitry Pavlov who does not share my views but supports my activity. The colleagues from the University of civil aviation (Department of Physics headed by Valery Arbuzov) took from me a part of my current job in spring semester. I am indebted to Edwin Taylor who long time ago asked me for the comments to the manuscript of his wonderful book 'Scouting the Black Holes' (written together with J.A.Wheeler), this book triggered the vague interest I had in this field before. Julia Hlynina helped me with the search in the electronic astronomical catalogues. And I would definitely be unable to cope with the editing problems in time without the help of Elena Ruchkova and especially of my elder kid George Laptev. I am really grateful to all these people.

Some of the results presented in the text were obtained during the work supported by the RFBR grants No. 07-01-91681-RA_a and No.08-02-01179a, and also by the grant HC No.149. 


\section{Contents}

Preface $\quad$ v

1. Classical relativity: Scope and beyond 1

1.1 Physics and mathematics: Long joint journey . . . . . . . . 1

1.1.1 From Pythagoras to Kepler and Newton . . . . . 1

1.1.2 Curvature, forces and fields . . . . . . . . . . 3

1.2 Inertial motion, relativity, special relativity . . . . . . 8

1.2.1 Bradley experiment . . . . . . . . . . . . . 10

1.2.2 Michelson and Morley experiment . . . . . . . 12

1.2 .3 Lorentz contraction . . . . . . . . . . . . . . . . . 18

1.2 .4 Special relativity . . . . . . . . . . . . . . . . 21

1.3 Space-time as a model of the physical world . . . . . . . 29

1.4 Generalized theory of relativity and gravitation . . . . . . 40

1.4.1 Tensors: who and why . . . . . . . . . . . . 40

1.4.2 Maxwell identities . . . . . . . . . . . . . . . 43

1.4.3 Least action principle . . . . . . . . . . . . . . . . . 48

1.4 .4 Mass and energy . . . . . . . . . . . . . . 51

1.4 .5 Field equations . . . . . . . . . . . 53

1.4.6 Gravitational waves . . . . . . . . . . 56

1.5 GRT - first approximation - predictions and tests . . . . 59

1.5.1 Newton gravity . . . . . . . . . . . . . 59

1.5.2 Classical tests . . . . . . . . . . . . . . 62

1.5.3 Gravitational lenses in GRT . . . . . . . . . . 66

1.6 Exact solutions . . . . . . . . . . . . . . 68

1.6.1 Star: static spherically symmetric case . . . . . 68

1.6.2 Universe: cosmological constant and expansion . . 71

1.7 Observations on the cosmological scale . . . . . . 75 
1.7.1 Rotation curves and their interpretation . . . . 77

1.7.2 Break of linearity in Hubble law . . . . . . . . 83

2. Phase space-time as a model of physical reality 85

2.1 Preliminary considerations . . . . . . . . . . . . . 85

$2.1 .1 \quad$ Scales . . . . . . . . . . . . . . . . 85

2.1.2 Boundaries . . . . . . . . . . . . . . . 89

2.1.3 Newton and Minkowski models for the intuitive space and time . . . . . . . . . . 95

2.2 Interpretation dilemma, variation principle, equivalence principle . . . . . . . . . . . . . . 101

2.2.1 Dilemma: new entity or new equations . . . . . 102

2.2.2 Comparison of methods . . . . . . . . . . . 106

2.2.3 On the variation principle . . . . . . . . . . . 109

2.2.4 On the equivalence principle . . . . . . . . . . 113

2.3 Construction of the formalism . . . . . . . . . . . . 117

2.3.1 Space and metric . . . . . . . . . . . 117

2.3.2 Generalized geodesics . . . . . . . . . . . . . 120

2.3.3 Anisotropic potential . . . . . . . . . . . . . . 122

2.3.4 Field equations . . . . . . . . . . . . . . . 124

2.3.5 Back to Einstein method . . . . . . . . . . . . 130

2.4 Gravitation force in anisotropic geometrodynamics . . . . 132

2.5 Model of the gravitation source and its applications . . . 140

2.5.1 Center plus current model . . . . . . . . . . . 140

2.5.2 Flat rotation curves of spiral galaxies . . . . . . 143

2.5.3 Tully-Fisher and Faber-Jackson relations . . . . . 145

2.5.4 Logarithmic potential in spiral galaxies . . . . . . 147

2.5.5 Classical tests on the galaxy scale . . . . . . . 148

2.5.6 Gravitational lenses in AGD . . . . . . . . . 156

2.5.7 Pioneer anomaly . . . . . . . . . . . . . 159

2.6 Electrodynamics in anisotropic space . . . . . . . . . . 160

2.6.1 Weak deformation of locally Minkowski metrics . 162

2.6.2 Lorentz force . . . . . . . . . . . . . . . . . 163

2.6.3 New term - "electromagnetic" vs. "metric" . . . . 166

2.6.4 Currents in anisotropic spaces . . . . . . . . . 168

2.7 Approaching phase space-time . . . . . . . . . . . 171

2.7.1 Coordinate-free dynamics . . . . . . . . . . . . 171

2.7.2 Generalized Lorentz transformations . . . . . . . . 172

2.7.3 Geometry, groups and their contractions .... 175 
2.8 Cosmological picture . . . . . . . . . . . . . . . 179

3. Optic-metrical parametric resonance - to the testing of the anisotropic geometrodynamics

3.1 Gravitation waves detection and the general idea of opticmetrical parametric resonance . . . . . . . . . . . 185

3.1.1 Space maser as a remote detector of gravitation waves . . . . . . . . . . . . . . . 193

$3.1 .2 \quad$ Atomic levels . . . . . . . . . . . . . . . . 196

3.1.3 Eikonal . . . . . . . . . . . . . . . . . . 199

3.1.4 Motion of a particle . . . . . . . . . . . 202

3.2 OMPR in space maser . . . . . . . . . . . . . . 205

3.3 Astrophysical systems . . . . . . . . . . . . . . . 214

3.3.1 GW sources . . . . . . . . . . . . . . . 215

3.3.2 Space masers . . . . . . . . . . . . . . . . . 217

3.3.3 Distances . . . . . . . . . . . . . . . . . . . . . . . 219

3.3.4 Examples . . . . . . . . . . . . . . . 222

3.4 Observations and interpretations . . . . . . . . . 225

3.4.1 Radio sources observation methods . . . . . . . . 225

3.4.2 Ultra-rapid variability and signal processing . . . 228

3.4.3 Search for the periodic components in space maser signals . . . . . . . . . . . . . . 231

3.5 On the search for the space-time anisotropy in Milky Way observations . . . . . . . . . . . . . . . 237

3.5.1 Mathematical formalism and basic equations . . . 238

3.5.2 Weak anisotropic perturbation of the flat

Minkowski metric . . . . . . . . . . . . . . 242

3.5.3 Modification of the OMPR conditions . . . . . . . 244

3.5.4 Investigations of the space-time properties . . . 246

Appendix A Optic-mechanical parametric resonance 249

A.1 Brief review . . . . . . . . . . . . . . . . . . . . 249

A.1.1 Mono-chromatic excitation . . . . . . . . . . . 249

A.1.2 Poly-chromatic excitation . . . . . . . . . . 250

A.1.3 Bichromatic fields . . . . . . . . . . . . . . . 251

A.1.4 Mechanical action on atoms . . . . . . . . . . 252

A.2 Force acting on a two-level atom . . . . . . . . . 255

A.2.1 Dynamics of an atom in the bichromatic field . . 256 
A.2.2 Stationary dynamics of atom . . . . . . . . 258

A.2.3 Light action on an atom . . . . . . . . . . 262

A.2.4 Groups of atoms in optical-mechanical parametric resonance . . . . . . . . . . . . . . . 264

A.2.5 Velocity change due to the force action . . . . . 265

A.2.6 Main result . . . . . . . . . . . . . . . 266

A.3 Probe wave absorption . . . . . . . . . . . . . . 267

A.3.1 Problem and its solution . . . . . . . . . . . 268

A.3.2 Assumptions and demands . . . . . . . . . . 278

A.4 Fluorescence . . . . . . . . . . . . . . . . 280

A.4.1 Calculation of the fluorescence spectrum . . . . . 284

A.4.2 Driving the TLAs by the bichromatic radiation . 288

A.4.3 Assumptions, demands and possible applications . 290

A.4.4 Conclusion . . . . . . . . . . . . . . . . . 292

$\begin{array}{ll}\text { Bibliography } & 295\end{array}$

Index 